\title{
Impact of train model variables on simulated energy usage and journey time
}

\author{
P. Lukaszewicz \\ Aeronautical and Vehicle Engineering, KTH, Stockholm, Sweden
}

\begin{abstract}
Several train model input variables, such as running resistance, line voltage, adhesion, braking release time and braking gain time, are studied. An analysis is performed on how variations in the variables impact relatively on calculated energy usage and running time of trains. The study shows that for the calculation of energy usage the simulations are most sensitive to variations in running resistance, followed by line voltage, adhesion, braking release time and braking gain time. For the running time, the study shows that variation in mechanical rolling resistance and air drag has a relatively small influence provided that the tractive force is big enough. If the line voltage and adhesion, which affect here the tractive force, drop below certain levels the running time increases dramatically. The braking release and gain times have little influence on the running time. The results also show which variables should be paid extra attention to, when constructing a train model.
\end{abstract}

Keywords: train modelling, train data, sensitivity, power consumption, energy usage, running time, simulations, ERTS.

\section{Introduction}

The correctness of computed results of energy usage and running time of trains in a railway network is dependent upon the chosen train model and input data. Therefore it is of interest to examine quantitatively how much the results can differ from each other if the input data used by the same train model varies and which data should be paid extra attention to.

By means of sensitivity analysis, the impact of the following variables is studied for a SJ Rc4 loco hauled freight train: 
- Running resistance, which is the total force acting against the travel direction.

- Adhesion.

- Tractive force (due to variation in catenary voltage).

- Braking gain time, which is the time it takes to obtain the desired braking force, from when the driver starts braking.

- Braking release time, which is the time it takes to reduce the braking force to zero, from when the driver stops braking.

Section 2 describes the method and models. The results are presented in section 3 and are discussed in section 4.

\section{Method and models}

This sensitivity analysis on how variation in input data affects the final results on computed energy usage and running time is here performed by means of the Energy and Running Time Simulator, ERTS. ERTS is a simulation program developed by $\mathrm{KTH}$ and has verified models and data, versus full-scale measurements, of trains and drivers. The verification shows that the discrepancy between calculated and measured train energy usage is within the measurement error of approx. $2 \%$ [1].

The train models are detailed especially with respect to braking and tractive forces, electrical efficiency, running resistance, adhesion and slippage.

The driver models in ERTS are developed from full-scale measurements [2]. Observations were made on how real drivers are handling the trains especially with respect to track profile, signalling and type of train and service. The developed driver models, not included here, can drive a train as an average driver would drive, or drive in an optimised way with respect to energy usage or running time.

The driver model in this study is constant and set to drive the train strictly in accordance with the signalled speed. The acceleration is performed at maximal powering. Braking is performed as late as possible with respect to the braking ability which is set to $1 / 3$ of the maximal braking force of the train. This level of the braking ability is obtained from observations on how the trains are driven in reality. The models are described in [1].

\subsection{The train model}

The train model represents a loco hauled freight train of mixed consist.

The locomotive is of type SJ Rc4 and the tractive force diagram for two different catenary voltages and powering levels is shown in Figure 1, together with the tractive force limit, $F_{\alpha}$, due to adhesion as it is modelled in ERTS.

The calculated magnitude of the tractive force, $F_{w}$, takes into account the powering level, effect of speed, catenary voltage and the tractive force, $F_{\alpha}$, available with respect to adhesion. In this study, no wheel slippage is present. 


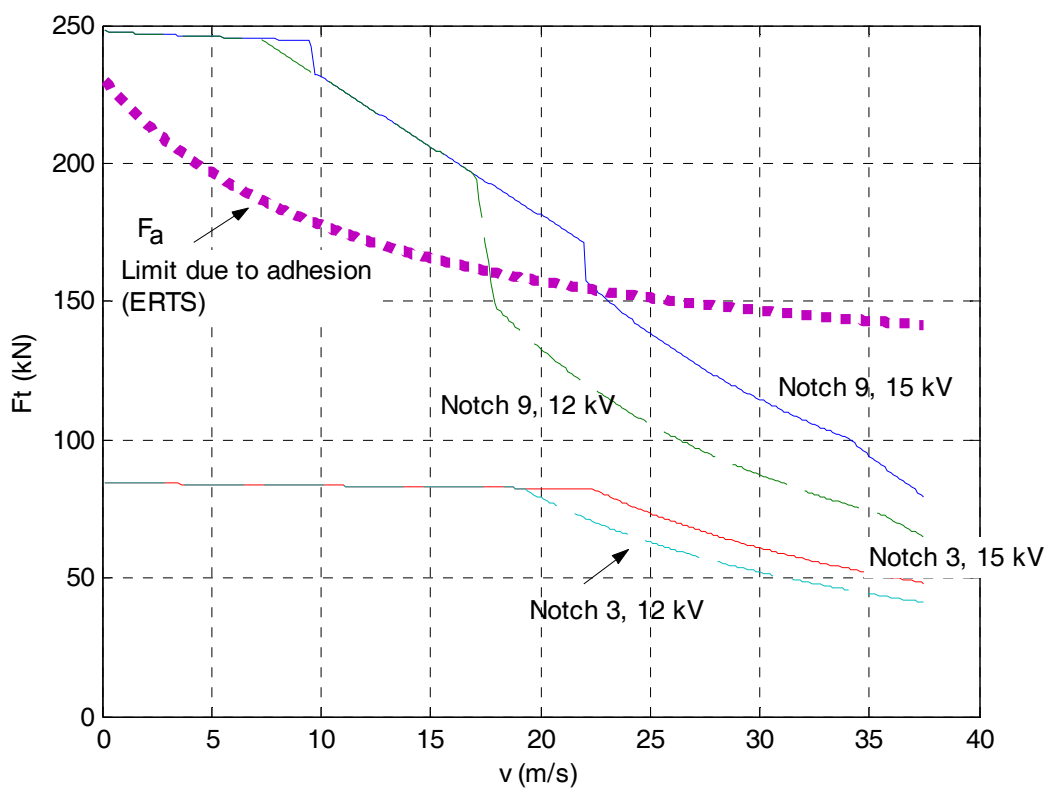

Figure 1: $\quad$ Tractive force diagram. Notch 9 is the maximal powering level.

This means that the train speed is the same as the tangential speed at the peripheral of the wheels of the locomotive. The tractive force at the wheels, is calculated by:

$$
F_{w}=\min \left(F_{t}, F_{\alpha}\right)
$$

The total energy usage, of the train is calculated at the pantograph level for two cases; $E_{1}$, when a tractive force is present and the train is moving, and $E_{2}$ when the train is coasting, braking or not moving.

$$
E_{\text {tot }}=\left\{\begin{array}{l}
E_{1}=\sum_{i=1}^{n} \frac{\left(F_{w(i)}+K_{j} a_{i}\right) v_{i}(1+\zeta) \Delta t_{i}}{\eta(p, v)_{i}} \frac{1}{3.6 \cdot 10^{6}} ; \quad v>0, \quad F_{w}>0 \\
E_{2}=\sum_{i=1}^{n} P_{0(i)} \Delta t_{i} ; \quad v=0 \text { or } F_{w}=0
\end{array}\right\}=E_{1}+E_{2}
$$

where, $E_{\text {tot }}$ is total energy usage in $\mathrm{kWh}, n$ is the total number of time steps $\Delta t$ during a simulation. $K$ is a constant accounting for the rotational masses, $a$ is the acceleration, $\zeta$ is the slippage $(=0), \eta$ is the efficiency of the locomotive as a 
function of power, $p$, and speed $v$, and $P_{0}$ is originating from the auxilliary power. The total running time is calculated from

$$
T=\sum_{n=1}^{i} \Delta t_{i}(\mathrm{~s}), \text { for } v>0
$$

The freight wagons in the train set have 2 axles/wagon and are of two types; open type Oms and covered type Hbis. Basic data for the test train is shown in Table 1:

Table 1: Nominal and basic data for the test train.

\begin{tabular}{|l|l|}
\hline Length, incl. loco & $418.5 \mathrm{~m}$ \\
\hline Mass, gross incl. loco & $1197 \mathrm{t}$ \\
\hline Mass of locomotive SJ Rc4 & $79 \mathrm{t}$ \\
\hline Axles, trailing & 52 \\
\hline Max speed & $100 \mathrm{~km} / \mathrm{h}, 27.8 \mathrm{~m} / \mathrm{s}$ \\
\hline Axle load, average & $21.5 \mathrm{t}$ \\
\hline Braking gain time, nominal & $15 \mathrm{~s}$ \\
\hline Braking release time, nominal & $30 \mathrm{~s}$ \\
\hline Braking level used & $1 / 3$ of $\max$ \\
\hline
\end{tabular}

The reason for choosing this train configuration is because of the existence of measured data [1] on energy usage, running resistance, tractive force, efficiency, braking ability and time lags in the tractive and braking systems.

\subsection{Track model}

The track model represents a tangent CWR. The length of the track is $88 \mathrm{~km}$. A simulation with nominal input data for the train model results in a running time of $3597 \mathrm{~s}$. The signalled speed restrictions are according to Table 2:

Table 2: Speed restrictions for the track model.

\begin{tabular}{|r|r|}
\hline Distance $(\mathrm{m})$ & speed $(\mathrm{km} / \mathrm{h})$ \\
\hline 0 & 100 \\
\hline 20490 & 40 \\
\hline 21364 & 100 \\
\hline 38152 & 70 \\
\hline 39288 & 100 \\
\hline 44106 & 70 \\
\hline 44566 & 100 \\
\hline 51322 & 40 \\
\hline 52534 & 100 \\
\hline 88000 & 100 \\
\hline
\end{tabular}




\section{Impact of variables on energy usage and running time}

\subsection{Simulation with nominal input data}

Figure 2 shows the speed profile for the train obtained from simulation with nominal input data. Table 3 shows the numerical results. This is the reference case, with which all other results are compared with in this study.

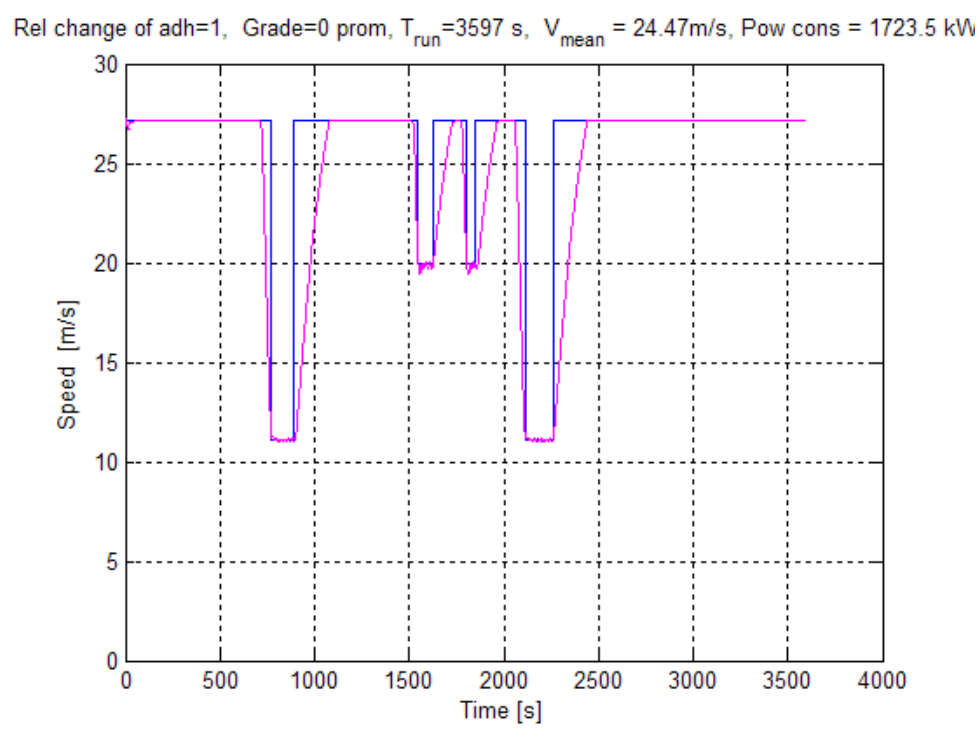

Figure 2: Speed profile from simulation with nominal input data.

Table 3: Results from simulation with nominal input data.

\begin{tabular}{|c|l|l|l|}
\hline Constant grade (\%o) & $E_{\text {tot }}(\mathrm{kWh})$ & $T(\mathrm{~s})$ & Mean speed $(\mathrm{m} / \mathrm{s})$ \\
\hline 0 & 1723.5 & 3597 & 24.47 \\
\hline 5 & 3329.9 & 3758 & 23.42 \\
\hline
\end{tabular}

\subsection{Running resistance}

The nominal running resistance, $F_{R 0}$, of the train set is obtained from full-scale measurements [1] and is calculated as a function of speed, $v$, by:

$$
F_{R 0}=11961+229.1 v+41.4 v^{2}
$$

The impact of variation of running resistance on energy usage and running time is shown in Figure 3. 


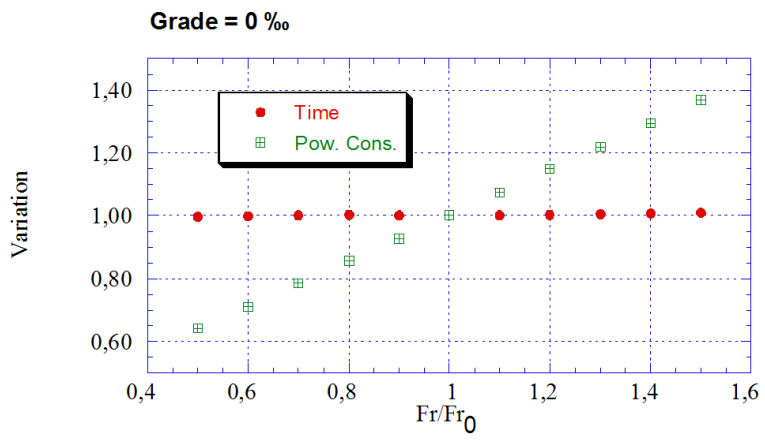

Figure 3: Impact of variation of running resistance on energy usage and running time.

In this case, the impact on running time is small, but big on the energy usage. If the resistance has large errors from input data together with resistance originating from grades, the tractive force of the locomotive might not be sufficient. In this case severe delays will be present.

\subsection{Adhesion}

The available nominal adhesion is calculated in ERTS by the Curtius-Kniffler formula [3] which has been modified [1] to better suit full-scale test data.

$$
\alpha_{0}=0.9\left(\frac{7.5}{44+3.6 v}+0.161\right)
$$

The results are shown in Figure 4. If the adhesion is higher than nominal, almost no variation occurs. However, if the adhesion ratio for this case starts decreasing below approx 0.7 , the running time starts increasing due to insufficient tractive power limited by the adhesion. Energy usage decreases mainly because of lower average speed which reduces the aerodynamic drag.

\subsection{Line voltage}

The tractive force of the locomotive SJ Rc4 is affected by the line voltage, see Figure 1. A voltage drop decreases the tractive force from the train speed of $17 \mathrm{~m} / \mathrm{s}$ and up.

The variation of running time and energy usage due to variation of line voltage is shown in Figure 5. The nominal voltage is $15 \mathrm{kV}$.

\subsection{Braking gain time}

The variation of braking gain time has for this studied case very small impact on the running time and energy usage, as shown in Figure 6. 

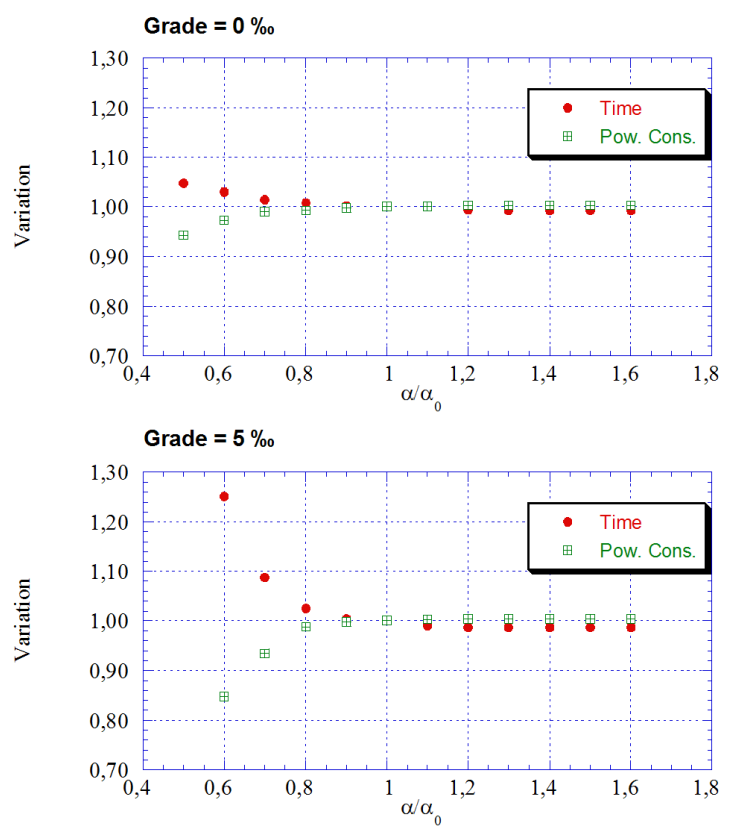

Figure 4: Impact of adhesion on energy usage and running time for grade 0 and $5 \%$.
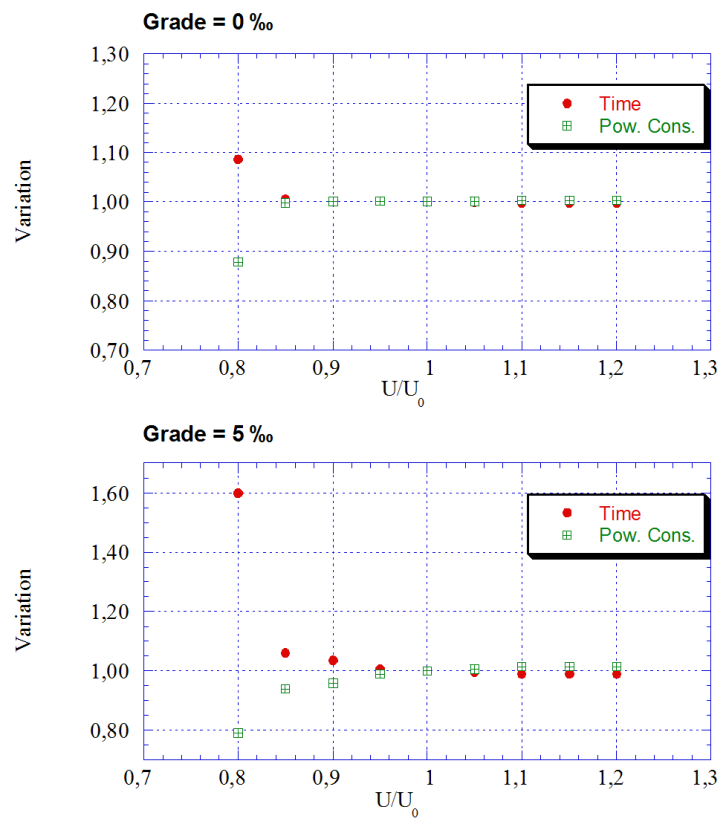

Figure 5: Variation of energy usage and running time due to variation of line voltage. 


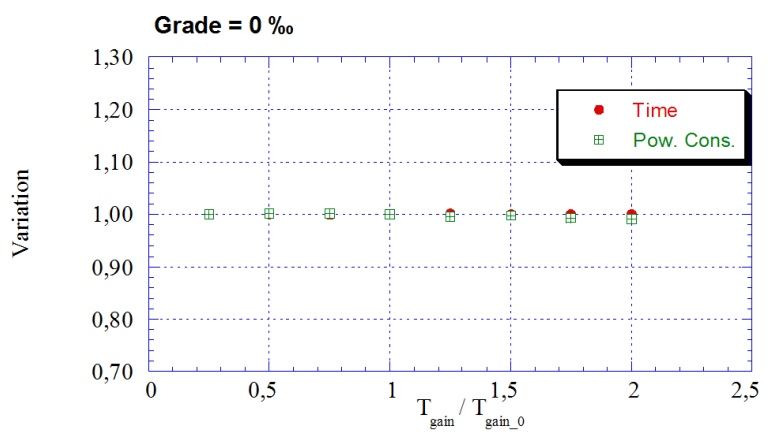

Figure 6: Variation of running time and energy usage due to variation of braking gain time.

\subsection{Braking release time}

The variation of braking release time has a slight impact on energy usage. If the braking release time is reduced, compared with the nominal $30 \mathrm{~s}$, a decrease in energy usage is distinguished, Figure 7.

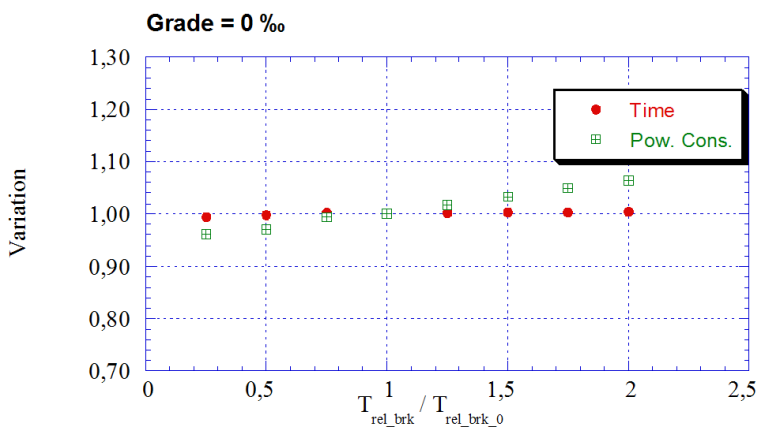

Figure 7: Variation of energy usage and running time due to variation of braking release time.

\section{Conclusions}

This study shows in a quantitative way the importance of choosing correct input data and their significance. It is therefore important to have up to date models, to collect train data, maintain databases and to have information on how and for which circumstances the data should be used.

Variation of running resistance has little effect on running time, provided the tractive force is sufficient. The energy usage is strongly dependent upon the running resistance.

When the available adhesion, as modelled in ERTS, drops under a certain level the energy usage drops as well. The running time increases significantly. 
When the line voltage drops and the tractive force is not sufficient, the energy usage drops as well. The running time increases significantly.

Variation of the braking gain and release times showed little significance in this study.

In this study, only the train model data is studied. An another important factor is the driver behaviour which has a strong impact on energy usage .

\section{References}

[1] Lukaszewicz P., Energy Consumption and Running Time for train. KTH Stockholm 2001. TRITA-FKT 2001:25. ISSN1103-470X.

[2] Lukaszewicz P., Driving describing parameters, energy consumption and running time. Computers in Railways VIII. Comprail 2002 Lemnos.

[3] Andersson, E. Berg, M.., Railway systems and vehicles (in Swedish). KTH 